\title{
État des lieux des mesures de prophylaxie sanitaire dans les élevages de poulets de chairs, souche COBB 500, dans le département de Korhogo (Côte d'Ivoire)
}

\author{
Gboko Konan Gatien BROU1, Moustapha DIABY1, Nahoulé SILUE1', Yadé René SORO1 \\ 1 Université Péléforo Gon Coulibaly de Korhogo, Enseignant-Chercheur, Institut de Gestion Agropastorale \\ (IGA), Laboratoire de Production et Santé Animale, BP 1223 Korhogo, République de Côte d'Ivoire. \\ Auteur correspondant ; E-mail : gatienbrou@gmail.com; Tél : (+225) 47156400 / 02870461
}

Original submitted in on $2^{\text {nd }}$ April 2018. Published online at www.m.elewa.org on $30^{\text {th }}$ June 2018 https://dx.doi.org/10.4314/jab.v126i1.9

\section{RESUME}

Objectif : Le présent travail évalue l'application des mesures de prophylaxie sanitaire dans les élevages de poulets de chairs, souche COBB 500, dans le département de Korhogo en Côte d'Ivoire.

Méthodologie et résultats : Trois cent (300) élevages ont ainsi été inspectés sur un cycle de production d'une durée de 45 jours. Les paramètres étudiés ont fait référence au profil sociologique des éleveurs ainsi qu'aux mesures de prophylaxie sanitaire. Sur la base de critères prédéfinis, quatre niveaux de prophylaxie sanitaire ont été définies allant du niveau mauvais au niveau excellent. II ressort que dans la zone d'étude, l'aviculture est majoritairement pratiquée par les hommes sans différence significative avec les femmes (test khi2, p-value $=0.4542>0,05$ ). Cependant, les hommes demeurent plus instruits (test khi2, $p$-value $=0.01573<0,05$ ). Relativement aux pathologies rencontrées dans les élevages, deux observations majeures peuvent être faites. Dans les cas de la maladie respiratoire chronique, la coccidiose et la salmonellose, un lien statistique a été observé entre leur apparition ou non et les niveaux de prophylaxie sanitaire. Les principales causes de leur apparition sont liées aux conditions de prophylaxie sanitaire mauvaise et médiocre. Dans le cas de la maladie de Gumboro, son apparition reste indépendante des conditions de prophylaxie sanitaire (test khi2, $p$-value $0.7596>0,05)$.

Conclusion et application des résultats : II ressort donc que le niveau de prophylaxie influence l'apparition de certaines maladies excepté la maladie de Gumboro qui nécessite en plus une vaccination. L'hygiène et la vaccination garantissent la bonne santé des oiseaux.

Mots-clés : Prophylaxie sanitaire, pathologie, poulets de chairs, Korhogo

\section{ABSTRACT}

Objective: The present work evaluates the application of sanitary prophylaxis measures in COBB 500 broiler chicken farms, in the Korhogo department of Côte d'Ivoire.

Methodology and results: Three Hundred (300) farms were inspected over a 45-day production cycle. The studied parameters referred to the sociological profile of the breeders as well as to the measures of sanitary prophylaxis. On the basis of predefined criteria, four levels of sanitary prophylaxis were defined ranging from bad to excellent. It appears that in the study area, poultry farming is mostly practiced by men without any significant difference with women (khi2 test, $p$-value $=0.4542>0.05$ ). However, men remain more educated (khi2 test, $p$-value $=0.01573<0.05$ ). With regard to the pathologies encountered in the farms, two major 
observations can be made. In the case of chronic respiratory disease, coccidiosis and salmonellosis, a statistical link was observed between their occurrence and levels of sanitary prophylaxis. The main causes of their appearance are related to poor sanitary prophylaxis conditions. In the case of Gumboro disease, its appearance remains independent of sanitary prophylaxis conditions (khi2 test, $p$-value $=0.7596>0.05$ ).

Conclusion and application of results: It is clear that the level of prophylaxis influences the appearance of certain diseases except Gumboro disease which also requires vaccination. Hygiene and vaccination guarantee the good health of the birds.

Keywords: Sanitary prophylaxis, pathology, broiler chickens, Korhogo

\section{INTRODUCTION}

L'aviculture joue un rôle très important dans le développement de nombreuses nations, tant pour des raisons nutritionnelles qu'économiques (Ndiaye, 2002). En Côte d'Ivoire, les productions avicoles contribuent pour $88 \%$ à la production de viandes et couvrent la demande d'œufs de consommation à hauteur de 100\% (FAO, 2008). La filière avicole contribue pour près de $2 \%$ au PIB national et pour près de $5 \%$ au PIB agricole (Boka, 2009). En 2015, elle a présenté plus de 240 milliards de chiffre d'affaire et créé 50000 emplois directs (Anonyme, 2014). L'élevage des volailles constitue ainsi, un maillon essentiel du système de production animale. Cependant, cette filière fait face à de nombreux problèmes qui perturbent son développement. II s'agit entre autres de la qualité et du coût des aliments, des problèmes liés à la commercialisation des poulets, mais surtout, des contraintes pathologiques entrainant de nombreuses mortalités (Bitty, 2013). En effet, plusieurs pathologies sont

\section{MATERIEL ET METHODES}

Présentation de la zone d'étude : Le département de Korhogo est situé au Nord de la Côte d'Ivoire, à $635 \mathrm{~km}$ d'Abidjan, avec une superficie de $12500 \mathrm{~km}^{2}$. II s'étend entre $5^{\circ} 16$ et $6^{\circ} 16$ de longitude Ouest et $8^{\circ} 32$ et $10^{\circ} 20$ de latitude Nord. Il est limité au Nord par la République du Mali, au Nord-est par le département de Sinématiali, au Nord-Ouest par les départements de Boundiali et observées dans les fermes avicoles ivoiriennes. Au niveau des poulets de chairs par exemple, les plus fréquentes sont la maladie respiratoire chronique, la colibacillose, la coccidiose et la maladie de Gumboro, (M'bari, 2000). Cette situation est due aux mauvaises conditions d'utilisation des vaccins, à l'utilisation de vaccins périmés, à la non vaccination des sujets ou aux mauvaises conditions hygiéniques des fermes (Dayon et Arbelot, 1997). L'une des méthodes de lutte contre l'apparition de ces maladies est l'application stricte des mesures de prophylaxies sanitaire et médicale (Izzedine, 2007). Ce sont entre autres, le nettoyage régulier des bâtiments et matériels d'élevage et le respect du calendrier de vaccination des poulets. Le présent travail a donc pour objectif de faire l'état des lieux de l'application des mesures de prophylaxies dans les élevages de volailles, souche COBB 500, du département de Korhogo.

Tingrela, au Sud par le département de Dikodougou, au Sud-ouest par le département de Séguéla et au Sud-est par le département de Niakara (Figure 1). Korhogo est la quatrième des villes ivoiriennes en termes de la taille de la population et d'importance des activités économiques (Anonyme, 2014). 


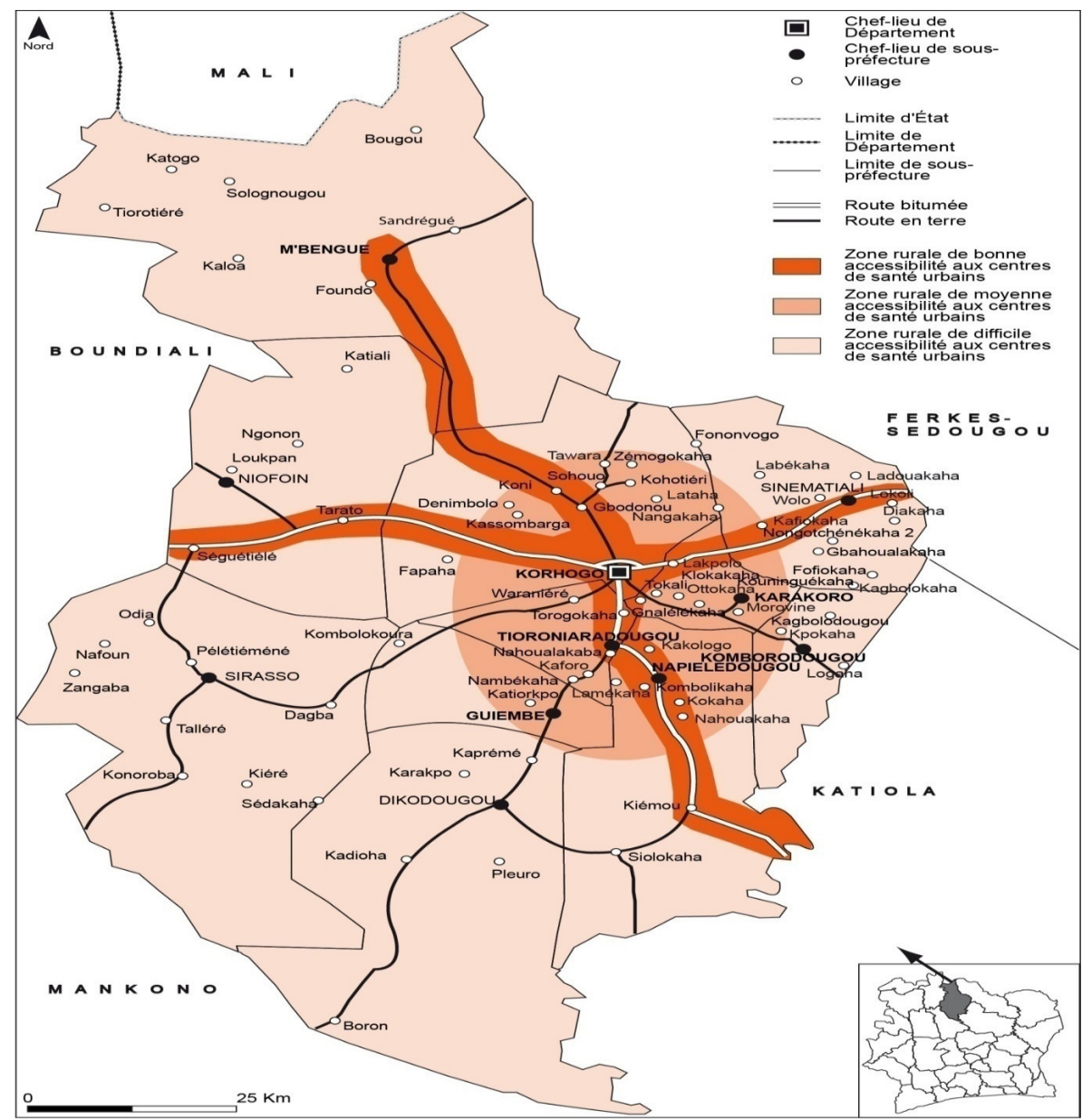

Figure 1: Carte du département de Korhogo (Anonyme, 2008)

: Limite de la zone d'étude et :fermes inspectées dans la zone d'étude

Matériel d'étude : Le matériel d'étude est constitué de fiches de questionnaires d'enquête. Les informations recherchées sont relatives au profil sociologique et aux mesures de prophylaxies sanitaire et médicale.

Méthode : L'enquête a été réalisée du 24 juillet au 24 Septembre 2017 auprès de 300 éleveurs de poulets de chairs, de souche Cobb 500, situés pour la plupart en périphérie de la commune de Korhogo. Cette enquête a été précédée d'une enquête exploratoire dans la période du 17 Juillet au 24 Juillet 2017. Cette dernière a permis de repérer l'étendue de la zone d'enquête et d'élaborer le type de questionnaire adéquat. Chaque éleveur a été visité une fois par semaine en vue de recueillir les informations relatives au profil sociologique et aux mesures de prophylaxies. Au niveau du profil sociologique, les informations recherchées ont concerné le sexe, l'âge, le niveau de formation et le niveau d'instruction. Quant aux mesures de prévalence, douze critères ont été déterminés. Ce sont, la distance entre deux fermes voisines, les normes de construction des bâtiments, la présence ou non de pédiluves et de clôture, la propreté de la ferme, le nettoyage journalier du matériel d'élevage, la présence d'autres animaux sur la ferme, l'incinération des poulets morts, le respect du vide sanitaire, l'existence d'une tenue de travail et enfin le respect du calendrier des vaccinations et traitements. Le respect d'une mesure de prévalence compte un point. Quatre niveaux de prophylaxie ont été définis. La mesure de prévalence est mauvaise, médiocre, bien et très bien lorsque l'éleveur totalise respectivement entre 0 et 3 points, entre 4 et 6 points, entre 7 et 9 points et enfin entre 10 et 12 points. Chez chaque éleveur, les maladies qui apparaissent sont notées.

Analyses statistiques : Le test de khi2 a été réalisé afin d'apprécier l'existence de différence significative au niveau des éléments du profil sociologique d'une part et entre les niveaux de prophylaxie et l'apparition des maladies. 


\section{RESULTATS}

Profils sociologiques des éleveurs

Genre et tranche d'âge : La tranche d'âge des éleveurs en fonction du genre est présentée par le tableau 1. Pour toutes les tranches d'âge, la proportion des hommes est supérieure à celle des femmes. La tranche d'âge dominante est celle comprise entre 30 et 45 ans avec 150 hommes et 24 femmes. Toutefois, le test de khi2 ( $p$-value $=0.4542>0,05)$ montre que statistiquement, les proportions des hommes et des femmes demeurent indépendantes des différentes classes d'âges.

Tableau 1 : Genre et tranches d'âge

\begin{tabular}{l|l|l|l}
\hline Tranche d'âge (année) & $\begin{array}{l}\text { Homme } \\
\text { Effectif (\%) }\end{array}$ & $\begin{array}{l}\text { Femme } \\
\text { Effectif (\%) }\end{array}$ & $\begin{array}{l}\text { Total } \\
\text { Effectif (\%) }\end{array}$ \\
\hline$(15-30)$ & $48(80 \%)$ & $12(20 \%)$ & $60(100 \%)$ \\
$(30-45)$ & $150(86,2 \%)$ & $24(13,8 \%)$ & $174(100 \%)$ \\
$(45$ et plus) & $54(81,8 \%)$ & $12(18,2 \%)$ & $66(100 \%)$ \\
\hline
\end{tabular}

Genre et niveau d'instruction : Le niveau d'instruction des éleveurs en fonction du genre est contenu dans le tableau 2. Le test de khi2 appliqué à ces paramètres indique un lien statistique entre eux (test khi2, $p$-value $=$ $0.01573<0.05$ ). Ainsi le tableau des résidus indique que les aviculteurs analphabètes et ceux du niveau primaire sont dominants chez les hommes, contrairement aux femmes chez lesquelles celles ayant les niveaux secondaire et universitaire sont majoritaires.

Tableau 2 : Niveau d'instruction des éleveurs en fonction du genre

\begin{tabular}{l|l|l|l|l}
\hline Genre & $\begin{array}{l}\text { Analphabète } \\
\text { Effectif (\%) }\end{array}$ & $\begin{array}{l}\text { Primaire } \\
\text { Effectif (\%) }\end{array}$ & $\begin{array}{l}\text { Secondaire } \\
\text { Effectif (\%) }\end{array}$ & $\begin{array}{l}\text { Universitaire } \\
\text { Effectif (\%) }\end{array}$ \\
\hline Homme & $72(92,3 \%)$ & $54(90 \%)$ & $66(78,6 \%)$ & $60(76,9 \%)$ \\
Femme & $6(7,7 \%)$ & $6(10 \%)$ & $18(21,4 \%)$ & $18(23,1 \%)$ \\
Total & $78(100 \%)$ & $60(100 \%)$ & $84(100 \%)$ & $78(100 \%)$ \\
\hline
\end{tabular}

Formation en aviculture et niveau d'instruction : La relation entre la formation en aviculture et le niveau d'instruction est contenue dans le tableau 3.La très faible valeur de la $p$-value après application du test de khi2 (test khi2, $p$-value $=4.864 \mathrm{e}-13<0,05)$ indique un fort un lien statistique entre ces paramètres. Le tableau complémentaire des résidus indique que la plupart des éleveurs analphabètes ou ayant un niveau d'instruction primaire n'ont aucune formation en aviculture. Ceux ayant reçu une formation en aviculture sont majoritairement les aviculteurs ayant des niveaux d'étude secondaire et universitaire.

Tableau 3 : Formation en aviculture et niveau d'instruction

\begin{tabular}{l|l|l|l|l}
\hline Formation & Analphabète & Primaire & Secondaire & Universitaire \\
\hline Formé & $20(26 \%)$ & $18(30 \%)$ & $63(75 \%)$ & $54(69 \%)$ \\
Non formé & $58(74 \%)$ & $42(70 \%)$ & $21(25 \%)$ & $24(31 \%)$ \\
Total & $78(100 \%)$ & $60(100 \%)$ & $84(100 \%)$ & $78(100 \%)$ \\
\hline
\end{tabular}

Pathologies rencontrées dans les fermes en fonction du degré de prophylaxie sanitaire

Maladie Respiratoire Chronique (MRC) : La maladie respiratoire chronique a été rencontrée dans toutes les fermes quel que soit le niveau de prophylaxie (tableau 4). Le test du khi2 ( $p$-value $=0.002395<0,05)$ indique donc un lien statistique entre l'apparition ou non de cette maladie et le degré d'application des mesures de prophylaxie. En outre, le tableau des résidus indique que les principales causes d'apparition de cette maladie sont liées aux conditions de prophylaxie sanitaire mauvaise et médiocre. 

poulets de chairs, souche COBB 500, dans le département de Korhogo (Côte d'Ivoire)

Tableau 4 : Apparition de la maladie respiratoire chronique

\begin{tabular}{l|l|l|l|l}
\hline & Mauvais & Médiocre & Bien & Très-Bien \\
\hline Oui & 18 & 90 & 42 & 6 \\
Non & 6 & 66 & 60 & 12 \\
\hline
\end{tabular}

Maladie de Gumboro : Comme la maladie respiratoire chronique, la maladie de Gumboro est apparue dans toutes les fermes comme l'indique le tableau 5 , et ce, quel que soit le niveau de prophylaxie en vigueur. Cependant, le test de khi2 montre qu'il n'existe aucune différence significative entre l'application des mesures de prophylaxie et l'apparition ou non de cette maladie dans les fermes $(p$-value $=0.7596>0.05)$. En d'autres termes, l'apparition de cette maladie reste indépendante des conditions de prophylaxies.

Tableau 5 : Apparition de la maladie de Gumboro

\begin{tabular}{l|l|l|l|l}
\hline & Mauvais & Médiocre & Bien & Très-Bien \\
\hline Oui & 6 & 48 & 36 & 6 \\
Non & 18 & 108 & 66 & 12 \\
\hline
\end{tabular}

Salmonellose : La salmonellose a été observée dans toutes les fermes comme mentionnée dans le tableau 6 . Une différence significative existe (test khi2, $\mathrm{p}$-value = $0.0268<0.05$ ) entre l'application des mesures de prophylaxie et son apparition dans les fermes. Le tableau des résidus montre cependant que les mauvaises conditions de prophylaxies favorisent l'apparition de cette maladie et contrairement lorsqu'elles sont meilleures.

Tableau 6 : Apparition de la salmonellose

\begin{tabular}{l|l|l|l|l}
\hline & Mauvais & Médiocre & Bien & Très-Bien \\
\hline Oui & 18 & 102 & 60 & 6 \\
Non & 6 & 54 & 42 & 12 \\
\hline
\end{tabular}

Coccidiose : Le tableau 7 indique la présence de la coccidiose dans les fermes en fonction du niveau de prophylaxie. Cette pathologie est observée dans des proportions importantes dans toutes les fermes quel que soit le niveau de prophylaxie appliqué. Le test de khi2 ( $p$ value $=0.01849<0.05$ ) indique donc un lien statistique entre l'application des mesures de prophylaxie et l'apparition de la coccidiose. Le tableau des résidus indique que les causes d'apparition de cette maladie sont principalement liées aux mesures de prophylaxies mauvaises ou médiocres.

Tableau 7 : Apparition de la coccidiose

\begin{tabular}{l|l|l|l|l}
\hline & Mauvais & Médiocre & Bien & Très-Bien \\
\hline Oui & 18 & 120 & 60 & 12 \\
Non & 6 & 36 & 42 & 6 \\
\hline
\end{tabular}

\section{DISCUSSION}

Le métier d'éleveurs de poulets de chair dans le département de Korhogo est pratiqué par les femmes et les hommes, ces derniers étant plus nombreux, quel que soit la tranche d'âge. Dayon et Arbelot (1997) font le même constat au Sénégal, où l'élevage moderne de volailles est une activité économique qui intéresse aussi bien les hommes que les femmes. Les femmes qui ont suivi une formation en aviculture s'installent de plus en plus à leur compte pour lutter contre la pauvreté. Au Bénin, les microfinances accompagnent les femmes dans la mise en place effective de leurs projets (Sodjinou,
2011). Dans le cadre de notre étude, les aviculteurs dont la tranche d'âge se situe entre 30 et 45 ans sont les plus nombreux. Cela pourrait s'expliquer par le fait que les individus ayant cette tranche d'âge ont déjà une activité principale et disposent de moyens pour financer l'élevage comme une activité secondaire (Bitty, 2013). II faut tout de même noter une différence tant au niveau d'instruction qu'au niveau de la qualification entre les hommes et les femmes exerçant ce métier. En effet, notre étude indique que les aviculteurs analphabètes et ceux du niveau primaire sont dominants chez les hommes contrairement 
aux femmes chez lesquelles, celles ayant les niveaux secondaire et universitaire sont majoritaires. Cette observation a été également faite à l'échelle nationale (FAO, 2008), indiquant que la plupart des hommes rencontrés dans ce secteur d'activité sont des déscolarisés ou n'ont jamais été à l'école. Comme explication, Oulon (2010) énonce l'ignorance et le coût de formation. En outre, la plupart des éleveurs analphabètes ou ayant un niveau d'instruction primaire n'ont aucune formation en aviculture. Ceux ayant reçu une formation en aviculture sont majoritairement les aviculteurs ayant des niveaux d'étude secondaire et universitaire. Contrairement aux hommes, la majeure partie des femmes pratiquant l'élevage de poulets de chair ont un bon niveau d'instruction. Comme explication, il faut noter qu'à la base, l'aviculture est considérée comme un métier réservé aux hommes (Boka, 2009). Les femmes qui s'y intéressent exercent cette activité après un parcours scolaire ou après une formation (Sodjinou, 2011). Leur objectif étant l'intérêt de bien se former pour mieux réussir l'activité (Ndiaye, 2002). Relativement aux pathologies rencontrées dans les élevages, deux observations majeures peuvent être faites. Dans les cas de la maladie respiratoire chronique, la coccidiose et la salmonellose, un lien statistique a été observé entre leur apparition ou non et les niveaux de prophylaxie sanitaire. Les principales causes de leur apparition sont liées aux conditions de prophylaxie sanitaire mauvaise et médiocre. En effet, dans le cas de la maladie respiratoire chronique, chez les jeunes oiseaux elle se manifeste par une détresse respiratoire, un manque d'appétit, une baisse de croissance et une augmentation de l'indice de consommation (Fettah, 2008). Chez les adultes, les symptômes les plus fréquents sont l'éternuement, la toux et une gêne respiratoire (ITAVI, 2009). L'apparition de la maladie respiratoire chronique ou aerosaculite est due à la présence d'énormes quantités de poussières dans les fermes lorsque les mesures de prophylaxie sanitaire sont mauvaises ou médiocres (Guérin et Boissieu, 2008). Cette maladie peut également apparaitre même quand les mesures d'hygiènes sont meilleures. Dans ce cas, les

\section{CONCLUSION}

Le profil sociologique des éleveurs varie d'une ferme à une autre. Les femmes sont présentes dans l'élevage des poulets de chair avec un niveau d'instruction et de formation acceptable par rapport aux hommes. La coccidiose et la salmonellose sont des maladies parasitaires dont l'apparition est liée aux conditions d'hygiène de la ferme. Les fermes qui ne sont pas touchées par ces maladies se caractérisent par la parents infectés transmettent le germe à leur progéniture par l'œuf (Khalen, 2013). Quant à la coccidiose, c'est une pathologie parasitaire intestinale dominante chez les poulets de chairs (Fettah, 2008). Les fermes qui disposent d'une bonne mesure de prophylaxie sanitaire sont moins exposées (Dosso, 2014). La transmission de cette maladie se fait par les fientes et litières infectées (Oulon, 2010). Les poulets atteints sont apathiques avec une diarrhée parfois sanguinolente et une forte mortalité (Dayon et Arbelot, 1997). Enfin, la salmonellose est une maladie infectieuse, contagieuse, inoculable qui touche les œufs, les poussins et les adultes (ITAVI, 2009). Les poussins âgés de moins de 3 semaines sont les plus touchés (M'bari, 2000). Les salmonelles sont toujours présentes dans le tube digestif. La transmission se fait d'une façon horizontale des adultes contaminés aux œufs et de façon verticale (Rabeson, 2010). Les fientes constituent les matières contaminants en souillant l'aliment, l'eau de boisson et le matériel d'élevage (Ndiaye, 2002). Chez les poussins elle provoque des signes de tristesses, de frilosité, d'anorexie et des ailes pendantes (Bitty, 2013). Dans le cas de la maladie de Gumboro par contre, le test de khi2 montre que l'apparition de cette maladie reste indépendante des conditions de prophylaxie sanitaire. Tous les éleveurs redoutent cette maladie qui peut décimer jusqu'à $60 \%$ de l'effectif des poulets et font correctement les vaccins (Izzedine, 2007 ; Guérin et Boissieu, 2008). Les mesures de prophylaxie médicale sont respectées par la quasitotalité des éleveurs contrairement aux mesures de prophylaxie sanitaire souvent négligées (M'bari, 2000). Cette maladie est très contagieuse et se propage rapidement d'un poulet à un autre par les fientes (Izzédine, 2007). Les vêtements et le matériel d'élevage contaminés assurent la transmission d'une exploitation à une autre (Fulbert et Alexandre, 2008). Les poulets âgés de 3 à 8 semaines sont les plus exposés (Rabeson, 2010). Les sujets malades sont apathiques, se blottissent les uns contre les autres, présentent de l'abattement, de l'anorexie, un ébouriffement des plumes avec diarrhée et déshydratation (Guérin et Boissieu, 2008).

propreté des bâtiments, du matériel d'élevage, de la litière, de l'eau et des aliments. La maladie respiratoire chronique est provoquée par d'énormes quantités de poussières dans les fermes. Elle apparait lorsque les bâtiments d'élevage ne sont pas suffisamment nettoyés et que la litière n'est pas régulièrement changée. La maladie de Gumboro survient dans les fermes lorsque les mesures de prophylaxie médicale et sanitaire sont mal 
appliquées. Le non-respect des mesures d'hygiène pourrait avoir des conséquences économiques désastreuses dans les fermes avec une faible rentabilité.

Khalen W. D. F., 2013. Étude de l'usage des antibiotiques dans les élevages avicoles modernes de la région de l'ouest du Cameroun. Thèse Méd.Vét., E.I.S.M.V., Dakar. $126 \mathrm{p}$

M'bari K. B., 2000. Contribution à l'identification des contraintes au développement de l'aviculture en Côte d'Ivoire. Thèse. Méd.Vét., E.I.S.M.V., Dakar. 101p.

Ndiaye M-L., 2002. Contribution à l'étude de la contamination microbiologique de la viande de volailles. Mémoire de DEUA, Faculté des sciences et techniques institut de technologie nucléaire appliquée I.T.N.A. Université Cheikh Anta Diop de Dakar.

Oulon E., 2010. État des lieux sur les mesures de biosécurité dans les fermes avicoles au Sénégal $:$ cas des départements de Rufisque et Thiès. Thèse : Méd. Vét., E.I.S.M.V., Dakar. 125p

Sodjinou E., 2011. Poultry-based intervention as tool poverty reduction and gender empowerment: Empirical evidence from Benin. PhD thesis, Institute of Food and Resource Economics Faculty of Life Sciences University of Copenhagen (Denmark). 255p.

Rabeson F. A., 2010. Enquête sérologique sur la maladie de Gumboro et biomoléculaire sur l'influenza aviaire hautement pathogène en aviculture traditionnelle au Sénégal. Thèse master II, E.I.S.M.V., Dakar. 41p 